\title{
Publisher's Note: Theory of Light Emission from Quantum Noise in Plasmonic Contacts: Above-Threshold Emission from Higher-Order Electron-Plasmon Scattering [Phys. Rev. Lett. 114, 126803 (2015)]
}

Kristen Kaasbjerg and Abraham Nitzan

(Received 2 April 2018; published 19 April 2018)

DOI: 10.1103/PhysRevLett.120.169902

This paper was published online on 26 March 2015 with incorrect labels in Figs. 3 and 4. Figures 3 and 4 have been replaced as of 9 April 2018. The figures are incorrect in the printed version of the journal; therefore, for the benefit of the print readership, the figures are replicated below.
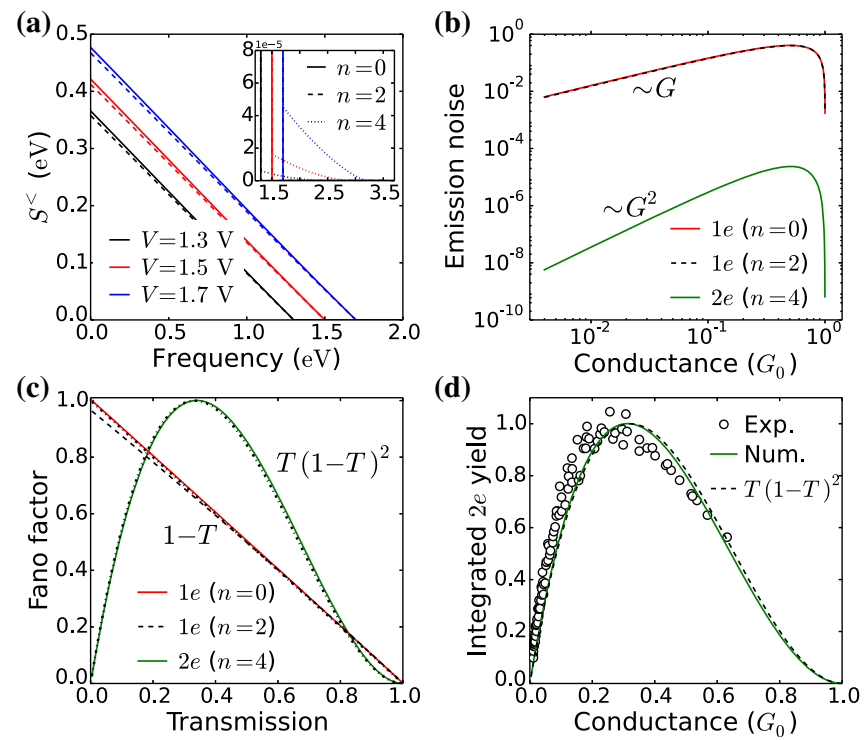

FIG. 3. (a) Noise spectrum to different orders in the $e$-pl interaction ( $n=0$, solid; $n=2$, dashed; $n=4$, dotted lines) for a contact with $T \sim 0.2$. The inset shows a zoom of the noise spectrum at $\omega \gtrsim e V$. (b), (c) Integrated $1 e$ and $2 e$ emission noise and Fano factors at $V=1.6 \mathrm{~V}$ vs conductance and transmission, respectively. The dash-dotted lines in (c) show the indicated analytic functions. (d) Integrated $2 e$ emission yield vs conductance at $V=1.6 \mathrm{~V}$. The circles show the experimental $2 e$ yield from Ref. [6]. In (c),(d), the Fano factors and yields have been normalized to unity at their maximum value (the $1 e$ Fano factors have been normalized with the $n=0$ maximum). Parameters: $\omega_{\mathrm{pl}}=1.5 \mathrm{eV}, \gamma_{0}=0.2 \mathrm{eV}, M=0.1, \Gamma_{\text {tip }}=10 \mathrm{eV}$.
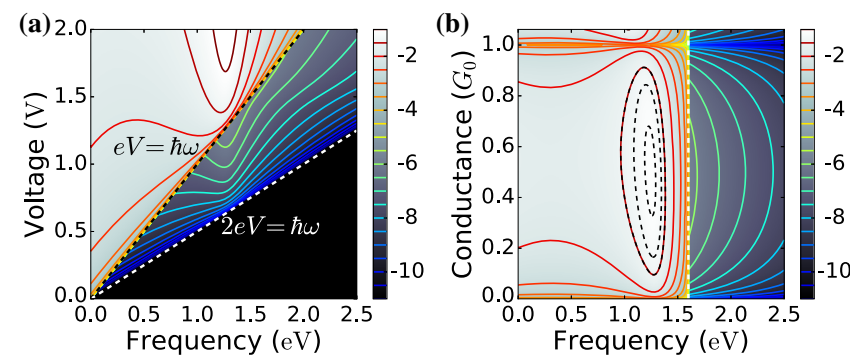

FIG. 4. (a) Emission spectrum vs applied voltage for a contact with $T \sim 0.2$. (b) Emission spectrum vs conductance at $V=1.6$ V. The plots show the emission rate $\Gamma_{\text {rad }}(\omega) \propto-\operatorname{Im} D^{<}(\omega)$ in units of $\gamma_{\text {rad }}$ on a logarithmic scale. Parameters: $\omega_{\mathrm{pl}}=1.5 \mathrm{eV}, \gamma_{0}=0.2 \mathrm{eV}$, $M=0.1, \Gamma_{\text {tip }}=10 \mathrm{eV}$. 